\title{
Determination of local heat transfer coefficient distribution on a vortex enhanced finned-tube heat exchanger fin using infrared thermography
}

\author{
by D. Bougeard* ,S. Russeil* and N-Y François**
}

\author{
* Mines Douai, El, F-59500 Douai,France \\ Univ Lille Nord de France, F-59000 Lille, France, daniel.bougeard@mines-douai.fr \\ * Mines Douai, El, F-59500 Douai,France \\ Univ Lille Nord de France, F-59000 Lille, France, serge.russeil@mines-douai.fr \\ ** Valeo Thermal Powertrain, F-78320 La Verrière, France nicolas-yoan.francois@valeo.com
}

\begin{abstract}
An innovative vortex-enhanced finned-tube heat exchanger geometry combining winglet longitudinal vortex generators and deflectors guiding the flow in tube wake has been experimentally studied by means of an infrared-based experimental method that allows the local heat transfer coefficient evaluation over the fin. The coefficient distribution is determined by using a transient technique and by calculating the energy balance during the fin cooling. The calculation model takes into account radiation with the surrounding and lateral heat conduction into the material. Results of local heat transfer coefficient distribution are presented for different Reynolds number values. The experimental convective heat transfer fields are first compared with CFD numerical results. A comparative analysis of heat transfer rates vs the smooth fin geometry is then presented.
\end{abstract}

\section{Introduction}

Gas-liquid plate fin-and-tube heat exchangers are widely used in engineering applications such as air conditioning, gas heating, process cooling and automotive industry. Many techniques are known to enhance heat transfer on the air side and reduce its dominant thermal resistance. Some of these techniques are called main flow enhancement techniques [1] where the gross characteristics of the flow are altered through geometry changes as for louvered or wavy fins for example. An alternative technique consists in secondary flow enhancement where local flow structures, usually longitudinal vortices, are deliberately created. Longitudinal vortices for heat transfer enhancement have been widely studied by Fiebig [2,3]. Within that whole framework, an innovative vortex-enhanced finned-tube heat exchanger geometry combining longitudinal vortex generators and deflectors guiding the flow in tube wake has been proposed in [4]. In [5] Bougeard et al showed, using CFD analysis, that strong longitudinal vortices are created by turbulence promoters and an efficient mechanism of flow guidance in tube wake is achieved. In the present work, the heat transfer coefficient distribution of this innovative geometry is investigated using a transient infrared thermography based procedure. This experimental investigation method, developed in our laboratory, requires the temporal evolution of the whole fin surface temperature recorded by IR thermography [6]. This technique is first applied to a single plate finand-tube geometry and compared locally and globally with CFD results. Then the performances of the complex enhanced geometry are presented and results are compared to the smooth fin geometry by analysing local heat transfer coefficient distribution over the fin. This enables to discuss the heat transfer enhancement techniques efficiency.

\section{Experimental set up and procedure}

\subsection{Test bench description}

The present experiments were conducted in an open-circuit wind tunnel, see Fig. 1. The upstream part of the wind tunnel has a section with airflow conditioning elements (fibreglass screen, honeycomb flow straightener and converging section) to supply an incoming airflow with uniform cross-sectional distribution and low turbulent level at the inlet of the test section located immediately downstream. Moreover the air temperature in the enclosed upstream chamber is controlled, using an air-conditioning unit, to provide a constant inlet air temperature at the entry of the test section. A rectangular observation window has been air-tightly set into the top-wall of the test section right above the plate fin and tube assembly to allow the measurement of the fin surface temperature. The axial airflow in the wind tunnel is driven by a fan located downstream and its volumetric flow rate is determined using a micro-manometer measuring the pressure drop across an orifice plate flowmeter. A long wave IR camera (AGEMA $®$ Thermovision 900 ) with a $10^{\circ}$ lens is placed at its minimal focalisation distance right above the heat exchanger model which surface has been thinly coated with black coating having high emissivity (95\%). The aforementioned rectangular observation window being transparent to infrared radiations, quantitative thermography measurement of the fin surface temperature is then made possible. This window, made of Zinc Selenide, has a direct transmission factor equal to $98 \%$ for infrared wavelengths ranging from 8 to $12 \mu \mathrm{m}$. The non-interlaced frames recorded by the infrared camera at a frequency equal to $15 \mathrm{~Hz}$ are post processed by a specific high accuracy signal-conditioning unit (ADDELIE $\left.{ }^{\circledR}\right)$. Finally, for a high accuracy, an in-situ calibration of the 
camera was performed [6]. Moreover a specific digital image restoration technique was developed to enhance spatial resolution. Details on this restoration technique that uses a two dimensional Wiener filter can be found in $[7,8]$.
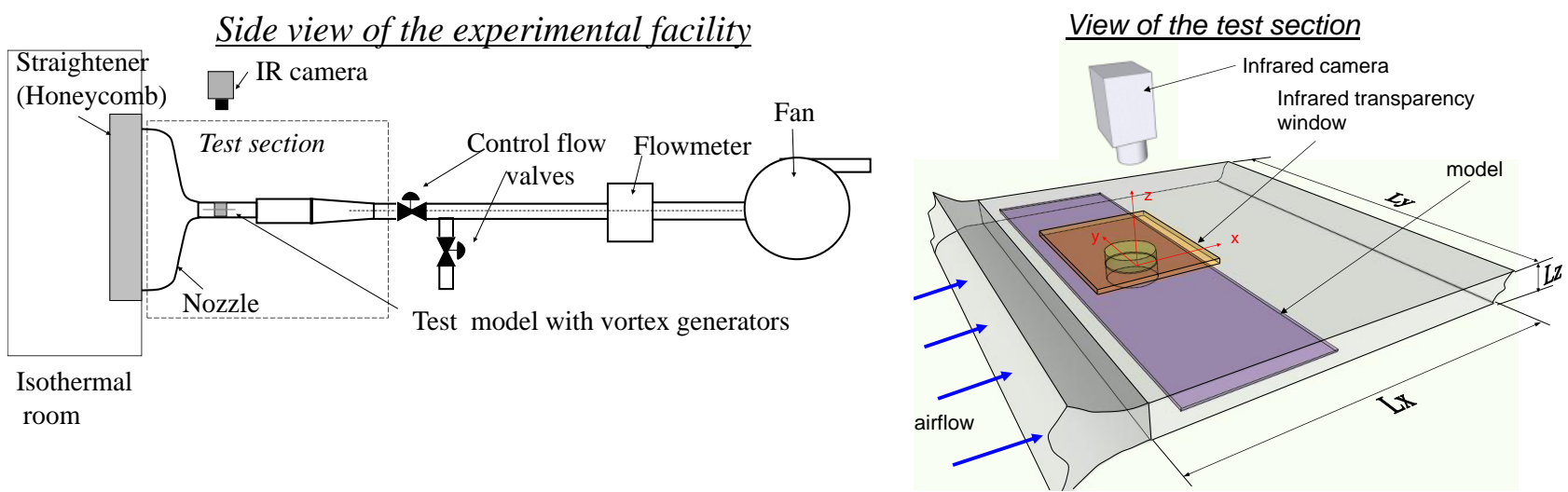

Fig. 1. Experimental facility

\subsection{Local heat transfer coefficient determination}

The experimental process is divided into two main steps. During the first period, a ceramic infrared emitter located right above the test model heats up uniformly the fin through the infrared transparent window, while no air flows throughout the test section. In the second phase, the IR emitter is removed, and the fin is suddenly cooled by incoming airflow. During the cooling period, the IR camera is used to record the temperature drop over the all fin surface. The sequential thermograms acquired are afterward post-processed with a specifically developed code, written using Matlab $^{\mathrm{TM}}$, to determine the local heat transfer coefficient distribution on the fin using the method developed in [7]. In this approach, the steady convective heat transfer coefficient distribution is deduced from the temperature temporal variation of the heat exchanger model fin surface during the transient cooling step. If we consider a thin plate element, at an initial temperature, which is suddenly cooled by forced convection, the heat balance can be written:

$$
\rho_{f} C_{p_{f}} e_{f} \frac{d T(x, y, t)}{d t}=\phi_{\text {conv }}+\phi_{\text {cond }}+\phi_{\text {rad }}
$$

with the right-hand side being the heat flux respectively transferred by convection, conduction and radiation. For the sake of simplification authors [9] often add the following assumptions: negligible heat conduction within the wall and negligible radiation heat transfer. Given this, the heat transfer coefficient can finally be derived from equation (1) after time integration between initial time $t_{\text {init }}$ (plate at uniform temperature) and final time $t_{\text {final }}$.

$$
h_{(x, y)}=\frac{\rho_{f} \cdot C_{p_{f}} \cdot e_{f}}{2\left(t_{\text {final }}-t_{\text {init }}\right)} \cdot \ln \left(\frac{T_{\text {ref }}-T_{\left(x, y, t_{\text {init }}\right)}}{T_{\text {ref }}-T_{\left(x, y, t_{\text {final }}\right)}}\right)=h_{\log (x, y)}
$$

The main advantage of this method which is usually referred to as the "log method", resides in its simplicity in the calculation of the local heat transfer coeffcicient: $h_{\log (x, y)}$.. Anyway, in case of strong local variation of the convection coefficient, neglecting the transversal conduction in the fin and using equation 2 can lead to important smoothing of the heat transfer coefficient distribution, cf. [6].

The method used in the present study consists in performing the time integration of Eq. (1) while taking into account the contributions of both conductive and radiation fluxes. Then it comes with the temperature T a function of $x, y$ and $t$ :

$$
\underbrace{\rho_{f} C_{p_{f}} e_{f} \int_{t_{\text {init }}}^{t_{\text {final }}(x, y)} \frac{d T}{d t} d t}_{l_{\text {total }}(x, y)}=h(x, y) \underbrace{\int_{t_{\text {init }}}^{t_{\text {final }}(x, y)}\left[T-T_{\text {ref }, \text { conv }}\right] d t}_{l_{\text {temp }}(x, y)}+\underbrace{\lambda_{f} e_{f} \int_{t_{\text {init }}}^{t_{\text {final }}(x, y)}\left(\frac{\partial^{2} T}{\partial x^{2}}+\frac{\partial^{2} T}{\partial y^{2}}\right) d t}_{l_{\text {cond }}(x, y)}+\underbrace{\varepsilon \int_{t_{\text {init }}}^{\varepsilon \int_{\text {final }}(x, y)}\left[T^{4}-T_{\text {ref }}^{4} \text {,rad }\right] d t}_{I_{\text {rad }}(x, y)}
$$

It's worth to note that in Eq. (3), the final instant may be different from one location $(x, y)$ on the fin surface to another one. Indeed it is determined in such a way that the temperature drop of each thin element of the fin is the same, that is the difference between the fin temperature at $a(x, y)$ location and the reference temperature, $\left[T\left(x, y, t_{\text {final }}\right)-T_{\text {ref, conv }}\right\rfloor$, has the same value for each possible location on the fin. Then for a given temperature difference $t_{\text {final }}$ is smaller for the points situated in the fin zones where the temperature decreases faster during the cooling period 
(leading edge zone, zone of the horseshoe vortex...) and higher in the other zones (wake zone for instance). Finally the local convective heat transfer coefficient at each location $(x, y)$ of the fin surface can be deduced from the simple formula given hereafter.

$$
h(x, y)=\frac{I_{\text {total }}(x, y)-I_{\text {cond }}(x, y)-I_{\text {rad }}(x, y)}{I_{\text {temp }}(x, y)}
$$

This method will be referred to as the "integral method" afterward.

\section{Single plate fin-and-tube heat exchanger model}

In [6] the experimental method was favourably compared to a developing plate flow experiment in a 2D channel configuration. In the present work a more complex three-dimensional flow is considered. Figure 2 presents the scaled-up finned-tube heat exchanger model under investigation. Its geometry consists in one flat plate with a circular disk fixed on each side of it. It is well known that a complex $U$ shaped tridimensional structure develops at the fin/tube junction. This 3D vortical flow that bends around the tube is the so-called Horse Shoe Vortex system. Fluid flow experimental investigations performed in our laboratory by PIV (Particle Image Velocimetry) have shown that the complex structure was formed of several vortices depending on Reynolds number and fin spacing $([10,11,12])$. We compare the experimental result with CFD simulation performed on the same geometry and for the same Reynolds Number to check the ability of the experimental method to capture local thermal characteristics. As reported on figure 2, the main geometric features are $\mathrm{D}, \mathrm{E}$ and $\mathrm{H}$ which respectively correspond to the tube diameter, the fin spacing and the tube centre location from the fin leading edge.

Top view of the test section

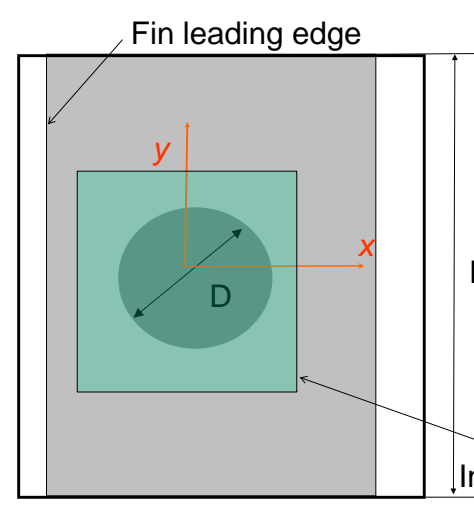

\section{Side view of the test section}

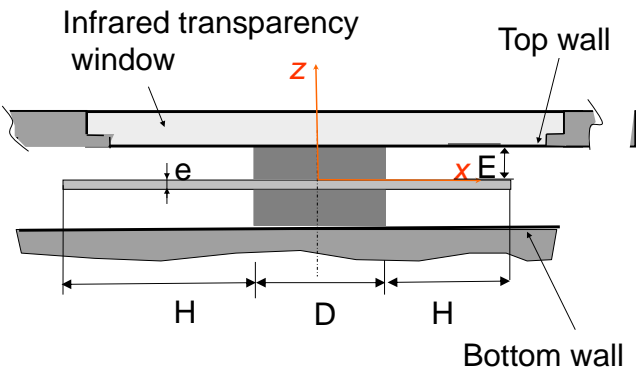

Fig. 2. Plate fin-and-tube heat exchanger model configuration

\subsection{Preliminary single finned-tube numerical simulation}

The heat exchanger model described above is numerically simulated using the CFD code Fluent. For a correct description of flow features and heat transfer coefficient, and for an accurate comparison with experimental investigations, the computational domain and the boundary conditions were both chosen as close as possible to the physical arrangement of the aforementioned experimental configuration. The Cartesian coordinates are also identical with the $x$-axis in the main flow direction, $z$-axis aligned with the tube axis and $y$ in the transverse directions. Details of this numerical investigation are not presented here, but only results which fall within the scope of the present work. Figure 3 illustrates the local heat transfer coefficient distribution on the fin surface around the tube base for $R_{D}=3320$. In accordance with previously published results, heat transfer coefficient values are found to be high in the vicinity of the fin leading edge as well as upstream the tube. On the contrary, low values are noticed downstream the tube because of low velocities magnitudes that prevail in this wake zone. High values of heat transfer coefficients that are encountered on the upstream portion of the fin are attributable to the thin boundary layer that is just starting to develop from the fin leading edge. As in classical developing channel flows the incoming boundary layer grows further downstream the fin leading edge, and to its increasing height corresponds a gradually decrease in heat transfer coefficient. This diminishing trend is then stopped thanks to the counteraction of the horse shoe vortex system which positive effect on heat transfer can be observed through high heat transfer coefficient values noticeable in a "U-shape" region wrapping round the tube. These thermal imprints on the fin surface are obviously linked to the horseshoe vortex structure which is found to be composed of two main vortices: see figure 3 (a) and 3 (b). 


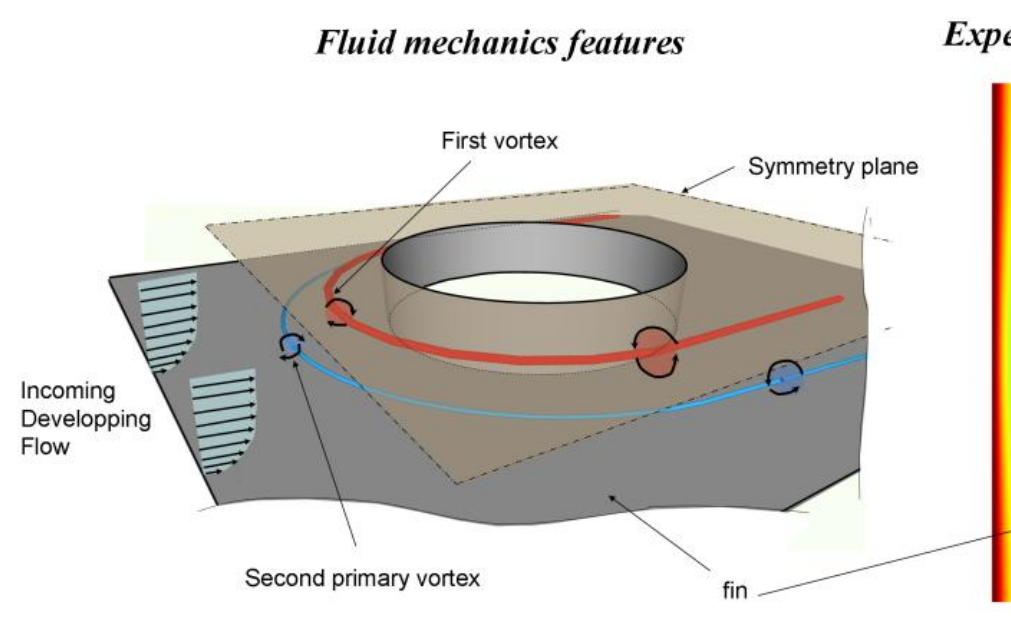

(a)
Expected heat transfer characteristics

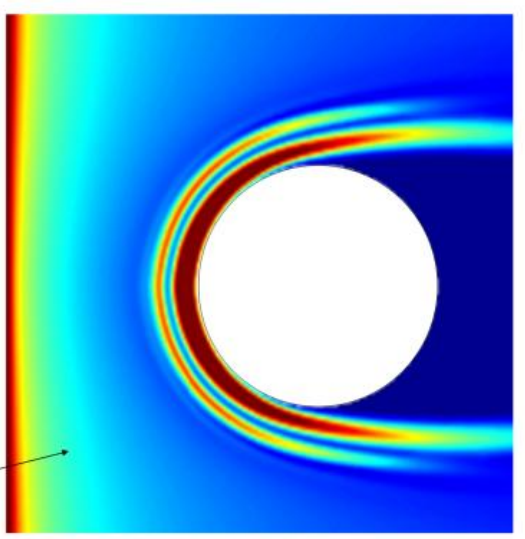

(b)

Fig. 3. Flow structure and heat transfer in a plain fin-and-tube geometry: (a) illustration of the horseshoe vortex formation around the tube (b) qualitative distribution of local heat transfer coefficient on the fin surface.

\subsection{Comparison of experimental and numerical results}

Figure 4 provides the comparison between both experimental and numerical results. The comparison is presented in the zone upstream and around the tube and for three Reynolds numbers. The maps of local Nusselt number are presented in figure $4 a$ for the two methods of investigation for $\mathrm{Re}_{\mathrm{D}}=3320$ It can be clearly seen that two main vortices are noticed in both cases and that their successive angular positions around the tube are in excellent agreement. The deviation between Nusselt number local values is also found quite acceptable. To extend further the comparative analysis figure $4 \mathrm{~b}$ presents a comparison of Nusselt numbers averaged in this upstream zone for various Reynolds numbers, $\operatorname{Re}_{D}=[1400 ; 2280,3320]$. The agreement between the two sets of data is very satisfactory in trend as well as in magnitude. The plots show that the greatest discrepancy $(8.7 \%)$ is noticed for the lowest Reynolds number, $\mathrm{Re}_{\mathrm{D}}=1400$. However it is worth to note that this difference is of the same order of magnitude of the experimental method uncertainties. These very satisfactory results prove that the present experimental method is able to accurately distinguish thermal imprints of complex 3D vortex structures. This also demonstrates the advantage of the method taking into account in particular the conduction fluxes and using an image deconvolution filter that significantly improves the spatial resolution of the determination of distributions of exchange coefficients.

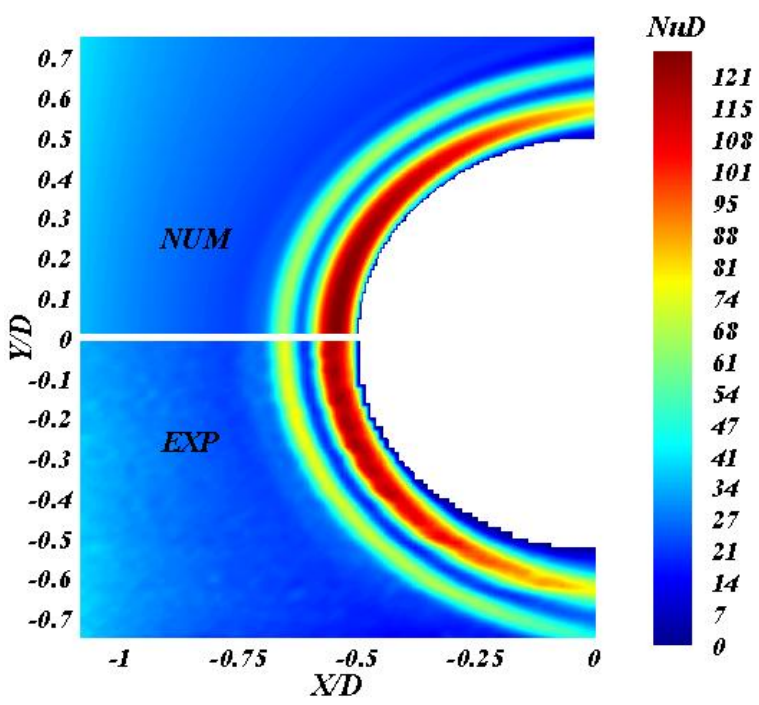

(a)

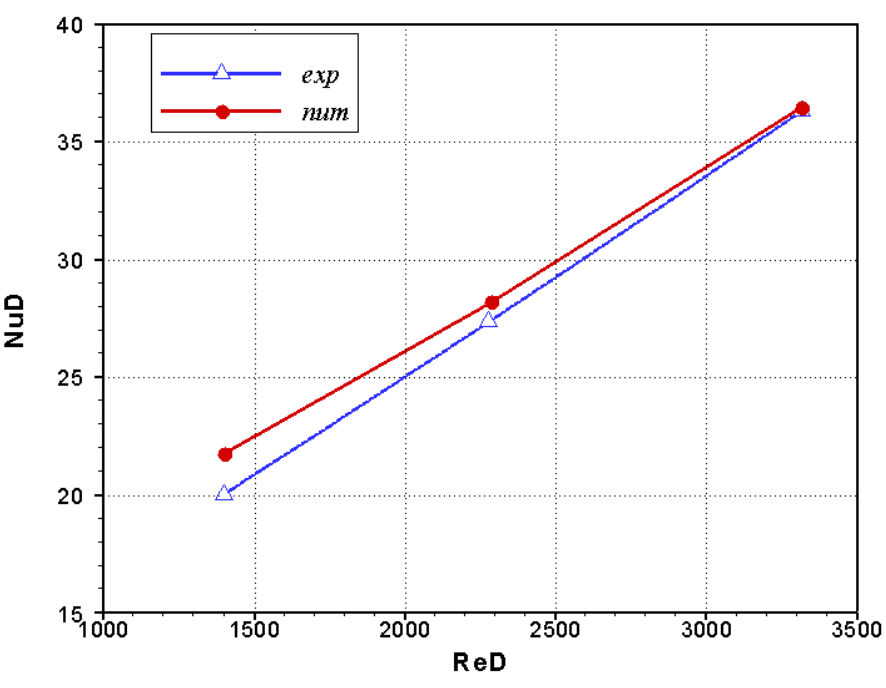

(b)

Fig. 4. (a) Local comparison of Nusselt number from numerical (CFD) investigation and experimental for $R e_{D}=3320$ - (b) Average Nusselt number evolution versus Reynolds number 


\section{Vortex enhanced finned-tube heat exchanger model}

\subsection{Geometry}

An innovative vortex-enhanced finned-tube heat exchanger geometry (referred to as $V E H X$ hereafter) has been proposed in [4]. As it can be seen from figure 5 (a) it is composed of a winglet-type vortex generator which is placed upstream the tube to create longitudinal vortices that develop downstream on both sides of the tube. A flow deflector is also located in the downstream vicinity of the tube with the main aim to guide the flow in the tube wake to reduce its size. The dimension and position of these two secondary flow enhancement elements are chosen as depicted in [4]. Previous numerical investigations, see [5], have shown that several rotational interacting flow structures are generated. A single tube row finned tube heat exchanger is considered in the present work.

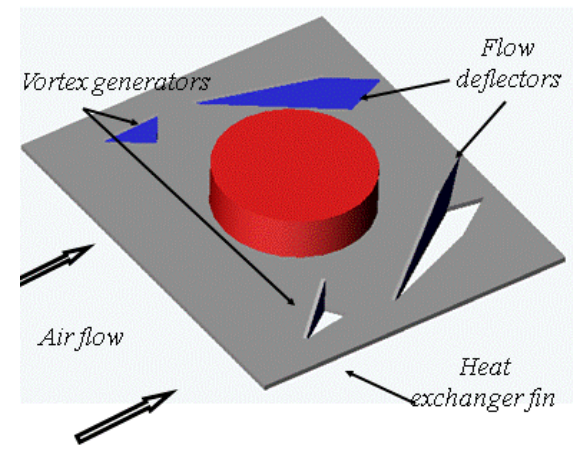

(a)
Upper view of the model

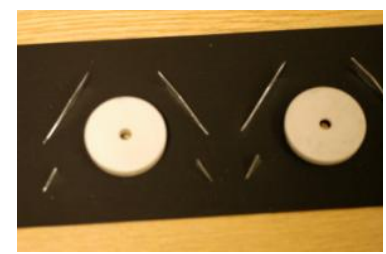

(b)
Side view of the experimental configuration

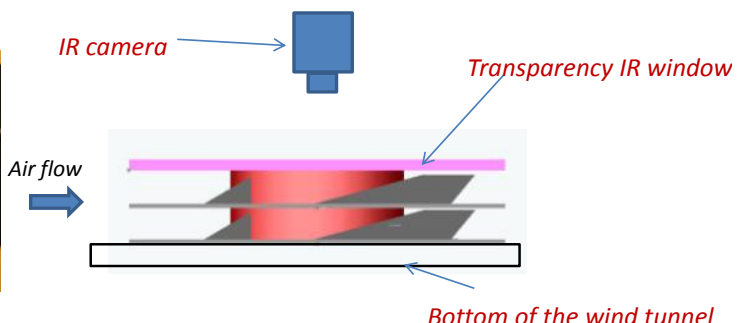

(c)

Fig. 5. (a) Vortex enhanced finned-tube geometry (b) Experimental model (c) Experimental configuration

To assess the VEHX performance in regards to a classical plain fin geometry experimental investigations are performed for two scaled-up models. The first one is one row smooth fin-and-tube assembly. The second one is the same one row fin-tube assembly with all the different vortex generators and flow deflectors fixed on the fin. It is worth to note that on this second model the vortex generators are not punched out of the fin (creating holes on the fin) as in the industrial patent but the vortex generators and flow deflectors are fixed on the fin, see figure 5 (b). Figure 5 (c) presents a side view of the VEHX model placed in the wind tunnel. In the experimental set-up, the fin is centered at the mid- height of the wind tunnel section. To ensure flow symmetry, vortex generators and flow deflectors are also set on the bottom wall of the wind tunnel.

\subsection{Results}

In a first step, we compare local distribution of heat transfer coefficient obtained by both of the two previously mentioned experimental methods. Figure 6 shows the Nusselt number for the VEHX geometry for the Reynolds number equal to 3840. Figure 6 (a) is obtained using Equation 2 of section 2 (log method). Figure 6 (b) is obtained using Equation 3 in section 2 (integral method), which takes account of the transverse conduction fluxes inside the fin. From the comparison it is obvious that the integral method gives a less blurry picture of the distribution of the Nusselt number over the whole fin. This sharper image (figure 6 (b)) enables to more clearly distinguish the various thermal imprints of the different vortex structures produced by the vortex generators and flow deflectors. This shows the advantage of the integral method for such investigations and its better ability to measure local variations in the convective heat transfer coefficient field.

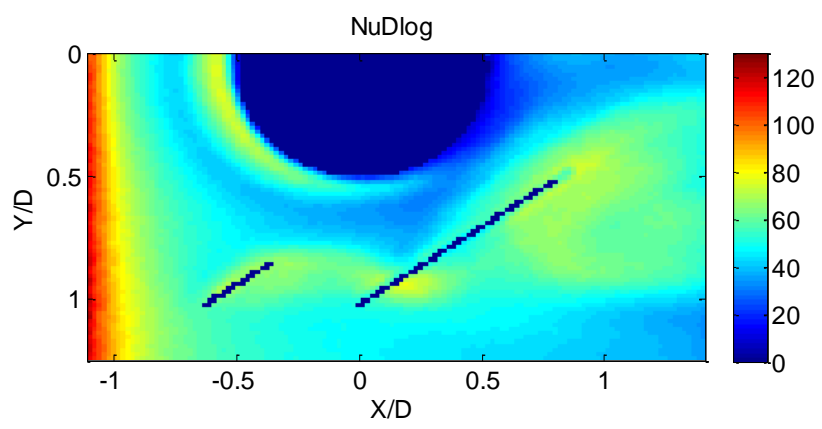

(a)

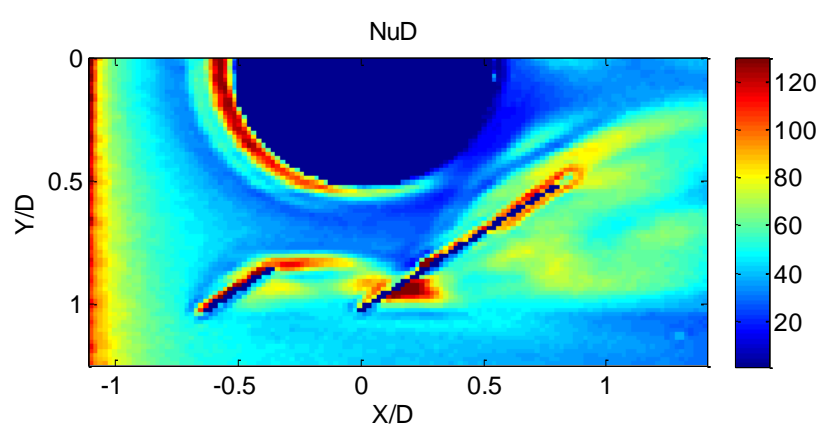

(b)

Fig. 6. (a) Nusselt number distribution with "log method" (b) Nusselt number distribution with "integral method" 
In a second step we determine the gain given by the improved geometry by comparing the VEHX geometry with the smooth one. Three Reynolds number values are considered. Unsurprisingly results show that when the Reynolds number increases the overall Nusselt number value over the fin also increases, see figure 7 . For both fin geometry the values of Nusselt number increase upstream and around the tube in U-shaped regions where the horse shoe vortex is influential. It can also be noticed that for high Reynolds number the HSV structures seem to be formed by two main vortices as expected. For the vortex enhanced geometry the thermal imprints show that the turbulence promoters create several vortices that significantly increase heat transfer rates. To better illustrate this, figure 8 (a) shows a zoom in the downstream vicinity of the vortex generator of the enhanced geometry. The two thermal imprints that are highlighted are directly linked to the corner vortex and the main vortex generated by such winglet-type vortex generator. Figure 8 (b) presents a zoom of the downstream vicinity the flow deflector. The several thermal imprints suggest the existence of a very complex flow structure with multiple interacting vortices.

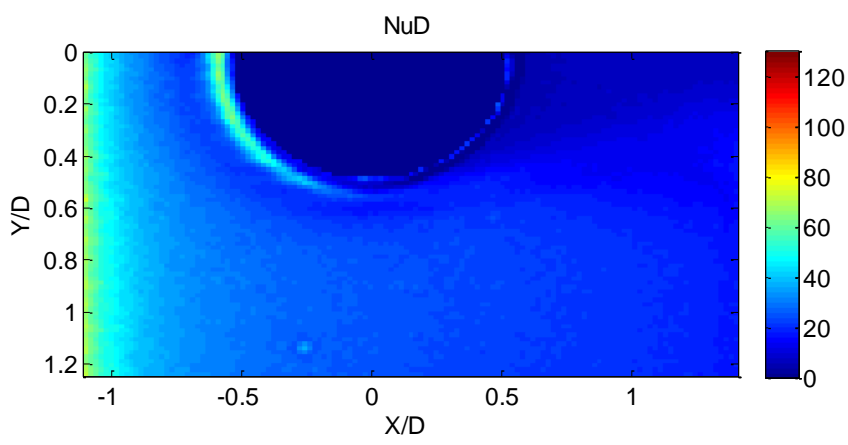

(a) $\mathrm{Re}=1400$

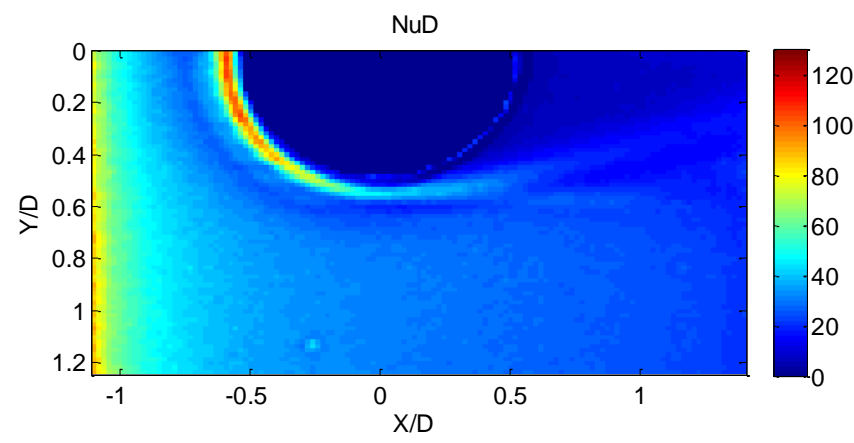

(c) $\mathrm{Re}=2280$

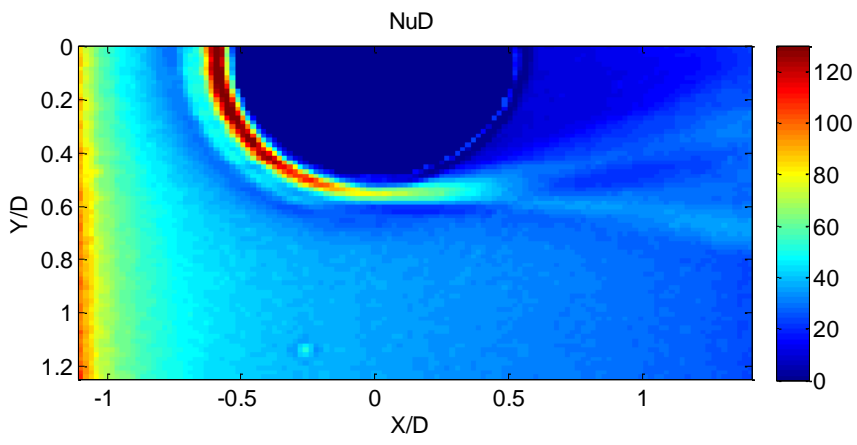

(e) $\operatorname{Re}=3320$

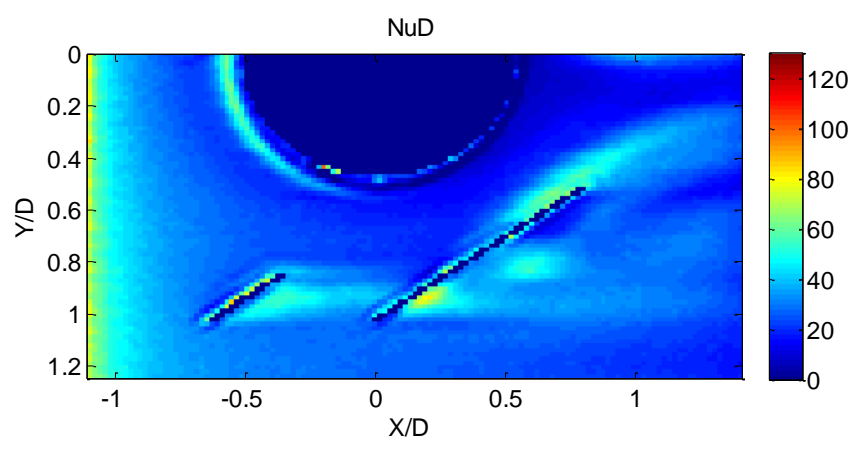

(b) $\mathrm{Re}=1400$

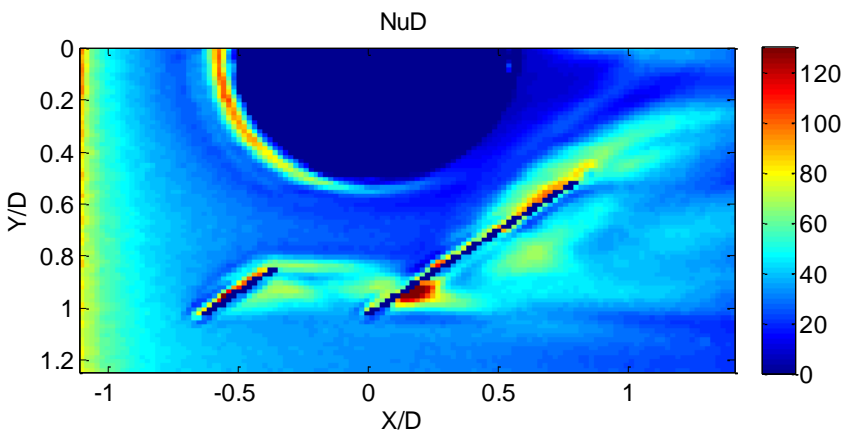

(d) $\operatorname{Re}=2280$

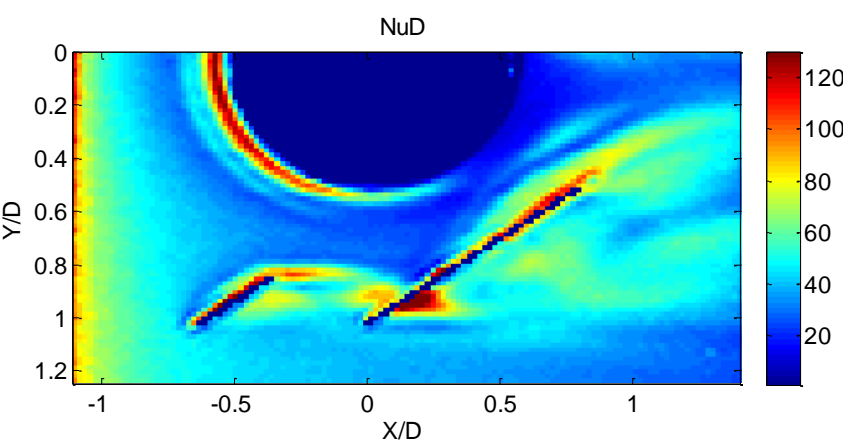

(f) $\operatorname{Re}=3320$

Fig. 7. Nusselt number distributions for the smooth fin (a), (c), (e) and the enhanced geometry (b), (d), (f) for $R e_{D}=1400,2280,3320$. 


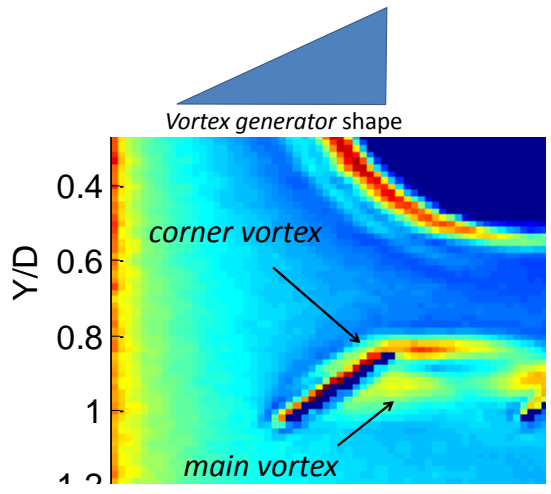

(a)

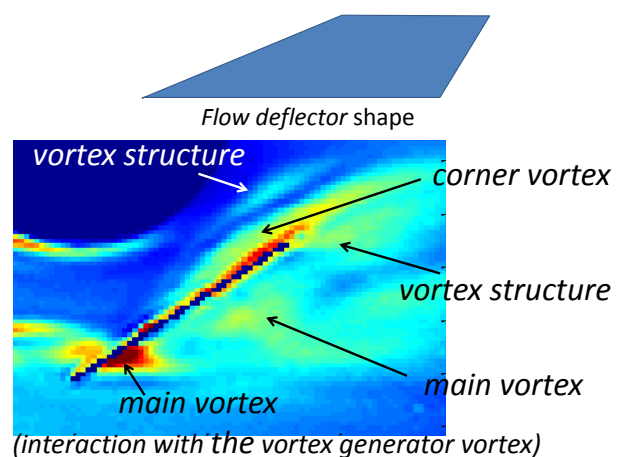

(b)

Fig. 8. Illustration of the multiple vortices interactions for the VEHX configuration.

Figure 9 shows the distribution of the ratio of Nusselt numbers for the vortex enhanced geometry and the smooth one $\left(\mathrm{Nu}_{\mathrm{D}} / \mathrm{Nu} \mathrm{DO}\right)$ for two Reynolds numbers. It can be seen that local enhancement of more than five hundred percents can be found on the fin surface. Most of fin regions that experience heat transfer augmentation are, as expected and previously mentioned, located downstream the vortex generator and the flow deflector. They correspond to secondary flows that are created due to adverse pressure gradients occurring upstream these obstacles that perturb the main flow. The guiding effect of the flow deflector is also clearly highlighted from the tube side towards the wake. Indeed a strong corner vortex develops upstream the deflector while the main flow is accelerated and deviated towards the tube wake. It is worth to note that the corresponding thermal imprints are found to be as efficient as the longitudinal secondary flows.
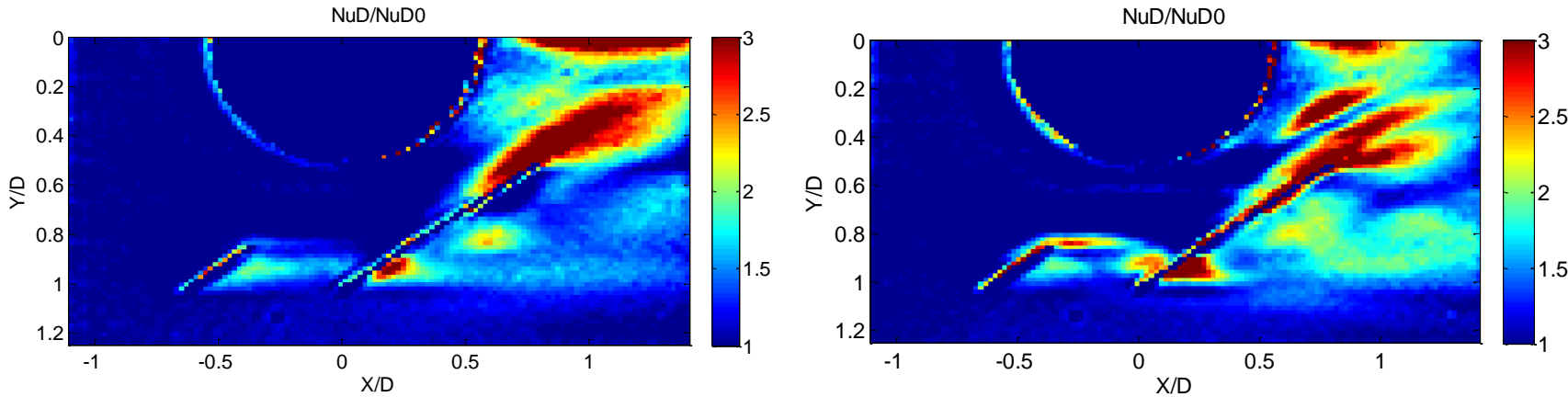

Fig. 9. Nusselt number enhancement factor (NuD/NuDO). On the left Reynolds number equal to 1400; on the right the Reynolds number is equal to 3320.

\section{Conclusions}

The present paper presents an innovative geometry of vortex enhanced plate finned-tube heat exchanger. This geometry combines vortex generators and flow deflectors guiding the main flow. Experimental investigations were made on two configurations: a smooth fin heat exchanger and the VEHX configuration. The performances of these two geometries were investigated using an infrared based transient method involving a scaled-up model that undergoes a transient cooling in a wind tunnel. The method presented in the present paper takes into account radiation with the surrounding and lateral heat conduction into the fin material.

The accuracy of the experimental method was first assessed on a single smooth plate fin-and-tube geometry by means of comparisons with CFD calculations. The results have evidently shown the ability of the method to determine precisely the heat transfer distribution. In particular, it has been shown that the spatial resolution of the technique is effective enough to precisely detect the thermal imprints of the Horse Shoe Vortex vortices that develop in the tube vicinity. The performances of the VEHX configuration were then investigated and compared to a single smooth fin-andtube heat exchanger. The results revealed that the new geometry promotes several fluid structures that greatly impact the convective fluxes. Locally, augmentations of more than 300 percents of the Nusselt number are found (vs the smooth fin configuration). The results also highlight the main flow path modification by the flow deflectors that guide the flow in the tube wake, and the efficiency of the longitudinal vortices generated by the vortex generators.

Future work will be devoted to compare the data provided by this experimental investigation to CFD calculation with the aim to help a better definition of the solver parameters (such as the turbulence model for example) in order get more reliable predictive CFD results. 


\section{REFERENCES}

[1] Jacobi A.M., Shah R.K., "Heat transfer surface enhancement through the use of longitudinal vortices: a review of recent progress". Experimental Thermal and Fluid Science, vol. 11(3), pp. 295-309, 1995.

[2] Fiebig M., "Embedded vortices in internal flow: heat transfer and pressure loss enhancement". International Journal of Heat and Fluid Flow, vol. 16(5), pp. 376-388, 1995.

[3] Fiebig M., "Vortices, Generators and Heat Transfer". Chemical Engineering Research and Design, vol. 76(2), pp.108-123, 1998.

[4] Magnier-Cathenod A.S., Russeil S., Bougeard D., "Fin with improved flow deflector and heat exchanger fitted with such a fin". Patent WO2007147754, 2007.

[5] Bougeard D., Russeil S., Simo Tala J.V., François N., "A vortex enhanced finned-tube heat exchanger with flow deflectors". Proceedings of Eurotherm 96 on Convective Heat Transfer Enhancement, paper 36, Brussels (Belgium), September 17-19, 2013.

[6] Bougeard D., "Infrared thermography investigation of local heat transfer in a plate fin and two-tube rows assembly". International Journal of Heat and Fluid Flow, vol. 28(5), pp. 988-1002, 2007,

[7] Bougeard D., Vermeulen J.P., Baudoin B., "Spatial resolution enhancement of an IR system by image restoration techniques". Proceedings of Eurotherm Seminar 42, Quantitative Infrared Thermography QIRT94, pp. 3-8, Sorrento (Italy), 23-26 August 1994.

[8] Bougeard D., Vermeulen J.P., Baudoin B., "Mesure du champ de température sur une ailette d'échangeur par thermographie infrarouge". Revue Générale de Thermique, vol. 34, pp. 325-334, 1995.

[9] Kim, Y. Y., Kim, K. S., Jeong, G. H., and Jeong, S. (2006). "An experimental study on the quantitative interpretation of local convective heat transfer for a plate fin and tube heat exchanger using the lumped capacitance method". International Journal of Heat and Mass Transfer, 49(1-2):230 - 239.

[10] Nacer-Bey M., Russeil S., Baudoin B., "Experimental study of the effect of flow velocity and fin spacing on the horseshoe vortex structure upstream of a one unit single-row plate-finned tube", Proceedings of Eurotherm 71 on Visualization, Imaging and Data Analysis in Convective Heat and Mass Transfer, pp. 163-168, Reims (France), 2002.

[11] Sanhaji M., Bougeard D., El-Abbadi A., S. Russeil, Nacer-Bey M., Baudoin B., "Infrared Thermography study of the local heat transfer coefficient in front of a single finned tube", 8th International Conference on Quantitative InfraRed Thermography, Cd-Rom paper n012, Padova (Italy), 2006.

[12] Simo Tala J.V., Russeil S., Bougeard D., Harion J.L., "Investigation of the flow characteristics in a multirow finned-tube heat exchanger model by means of PIV measurements". Experimental Thermal and Fluid Science, vol. 50, pp. 45-53, 2013. 